\title{
Dichotomy in temporal and thermal spin correlations observed in the breathing pyrochlore $\mathrm{LiGa}_{1-x} \mathrm{In}_{x} \mathrm{Cr}_{4} \mathrm{O}_{8}$
}

\author{
S. Lee ${ }^{1,2}$, S.-H. Do ${ }^{1}$, W. Lee ${ }^{1}$, Y. S. Choi ${ }^{1}$, J. van Tol ${ }^{3}$, A. P. Reyes $\mathbb{i D}^{3}$, D. Gorbunov ${ }^{4}$, W.-T. Chen $\mathbb{D}^{5,6}$ and K.-Y. Choi $\mathbb{D}^{7 凶}$
}

A breathing pyrochlore system is predicted to host a variety of quantum spin liquids. Despite tremendous experimental and theoretical efforts, such sought-after states remain elusive as perturbation terms and lattice distortions lead to magnetic order. Here, we utilize bond alternation and disorder to tune a magnetic ground state in the Cr-based breathing pyrochlore $\mathrm{LiGa}_{1-x} \mathrm{In}_{x} \mathrm{Cr}_{4} \mathrm{O}_{8}$. By combining thermodynamic and magnetic resonance techniques, we provide experimental signatures of a spin-liquid-like state in $x=0.8$, namely, a nearly $T^{2}$-dependent magnetic specific heat and persistent spin dynamics by muon spin relaxation ( $\mu \mathrm{SR}$ ). Moreover, ${ }^{7} \mathrm{Li}$ NMR, ZF- $\mu \mathrm{SR}$, and ESR unveil the temporal and thermal dichotomy of spin correlations: a tetramer singlet on a slow time scale vs. a spin-liquid-like state on a fast time scale. Our results showcase that a bond disorder in the breathing pyrochlore offers a promising route to disclose exotic magnetic phases.

npj Quantum Materials (2021)6:47; https://doi.org/10.1038/s41535-021-00347-0

\section{INTRODUCTION}

A pyrochlore lattice provides a fertile ground to discover exotic quantum and topological phenomena since a three-dimensional (3D) network of corner-sharing tetrahedra features a macroscopic ground-state degeneracy ${ }^{1-6}$. When quantum fluctuations are sufficiently strong, the pyrochlore Heisenberg antiferromagnet is predicted to harbor a spin-liquid ground state, whose defining characteristics are fractionalized excitations, emergent gauge fields, and long-range entanglement ${ }^{7-10}$. However, such a quantum spin liquid (QSL) in pyrochlore materials has proven exceptionally difficult to achieve because a large coordination number suppresses quantum fluctuations and a range of perturbations such as spin-lattice couplings is apt to stabilize magnetic order ${ }^{11-13}$.

In the context of a generalized pyrochlore lattice, a breathing pyrochlore has recently garnered much attention as a reservoir of exotic quantum states ${ }^{14,15}$. In the breathing pyrochlore, $A$ and $B$ tetrahedra alternate in size and, thus, exchange interactions (see Fig. 1a). As such, its spin Hamiltonian is given by $\mathcal{H}=J_{A} \sum_{i, j \in \mathrm{A}} \mathbf{S}_{i} \cdot \mathbf{S}_{j}+J_{B} \sum_{i, j \in \mathrm{B}} \mathbf{S}_{i} \cdot \mathbf{S}_{j}$. Here, the breathing anisotropy $B_{f}=J_{B} / J_{A}$ quantifies the degree of the bond alternation. By gauging inter-tetrahedral coupling $J_{B}$, decoupled tetrahedra $\left(B_{f}=0\right)$ is tuned to a regular pyrochlore $\left(B_{f}=1\right)$. At the endpoints, the ground states are tetramer singlet and $\mathrm{QSL}^{9,16}$. The breathing pyrochlore has an advantage over its uniform counterpart in searching for QSL thanks to its strong thermal stability ${ }^{17}$. Besides, the inclusion of magnetic anisotropies to a pyrochlore Heisenberg model leads to a realization of Weyl magnons-a bosonic analog of a Weyl fermion in electron systems and a fracton spin liquid whose elementary excitations mimic electric and magnetic fields having the form of rank-2 tensors ${ }^{18,19}$.

On the material side, the $\mathrm{Cr}$-based breathing pyrochlore $\mathrm{Li} A \mathrm{Cr}_{4} \mathrm{O}_{8} \quad\left(A=\mathrm{Ga}^{3+}, \mathrm{In}^{3+}\right.$; space group $\left.F \overline{4} 3 \mathrm{~m}\right)$ is a particularly interesting instance because $B_{f}$ can be tuned by varying the composition of $\mathrm{Li}$ and $A^{15}$. $\mathrm{LiGaCr}_{4} \mathrm{O}_{8}$, having the breathing anisotropy $B_{f}=0.6$, behaves much like a uniform pyrochlore. On the other hand, $\mathrm{LilnCr}_{4} \mathrm{O}_{8}$ has the small $B_{f}=0.1$, in which the $\mathrm{A}$ tetrahedra are weakly coupled through the inter-tetrahedral interaction $J_{B}$. Despite the large difference in $B_{f}$, both the $\mathrm{LiCr}_{4} \mathrm{O}_{8}$ compounds show commonly two-stage magneto-structural transitions at $T_{\mathrm{S}}=16(17) \mathrm{K}$ and $T_{\mathrm{M}}=13(14) \mathrm{K}$ for $\mathrm{A}=\ln (\mathrm{Ga})^{20}$. The lasting antiferromagnetic orders suggest that the bond alternation of the $x=0$ and $x=1$ compounds is not sufficient to repress the effect of spin-lattice coupling that is responsible for a cubic-totetragonal transition accompanying structural and magnetic phase segregation. In this situation, bond randomness can counteract the magnetoelastic transition by inhibiting cooperative lattice distortions. This raises the exciting prospect of unveiling concealed magnetic behaviors of the breathing pyrochlore lattice, not being interrupted by long-range magnetic order.

In this work, we investigate spin dynamics and low-energy excitations of the bond-disordered breathing pyrochlore $\mathrm{LiGa}_{0.2} \mathrm{In}_{0.8} \mathrm{Cr}_{4} \mathrm{O}_{8}$ (marked by the inverse triangle in Fig. $1 \mathrm{~b}$ ). By combining multiple magnetic resonances with thermodynamic techniques, we find a thermal and temporal dichotomy between a coupled tetramer singlet and a spin-liquid-like state with no indication of the substitution-induced phase coexistence. Spin fluctuations probed on the different time scales evidence that the QSL-like state is pertinent to an emergent diluted pyrochlore lattice by a random bond alternation.

\section{RESULTS \\ Magnetic phase diagram}

As sketched in Fig. $1 \mathrm{a}$, the $A$-site ordered $\mathrm{Li} A \mathrm{Cr}_{4} \mathrm{O}_{8}(A=\mathrm{Ga}, \mathrm{In})$ spinels are a nearly perfect realization of the breathing pyrochlore lattice due to the significant difference between the $\mathrm{Li}^{+}$and $A^{3+}$ valence states. The Ga-for-In substitution disrupts a uniform arrangement of the two alternating tetrahedra and leads to the rich magnetic phase diagram of $\mathrm{LiGa}_{1-x} \mathrm{In}_{x} \mathrm{Cr}_{4} \mathrm{O}_{8}$, as shown in Fig.

\footnotetext{
${ }^{1}$ Department of Physics, Chung-Ang University, Seoul, Republic of Korea. ${ }^{2}$ ISIS Neutron and Muon Source, STFC Rutherford Appleton Laboratory, Didcot, Oxfordshire, United Kingdom. ${ }^{3}$ National High Magnetic Field Laboratory, Florida State University, Tallahassee, FL, USA. ${ }^{4}$ Hochfeld-Magnetlabor Dresden (HLD-EMFL), Helmholtz-Zentrum DresdenRossendorf, Dresden, Germany. ${ }^{5}$ Center for Condensed Matter Sciences, National Taiwan University, Taipei, Taiwan. ${ }^{6}$ Center of Atomic Initiative for New Materials, National Taiwan University, Taipei, Taiwan. ${ }^{7}$ Department of Physics, Sungkyunkwan University, Suwon, Korea. ${ }^{凶}$ email: choisky99@skku.edu
} 

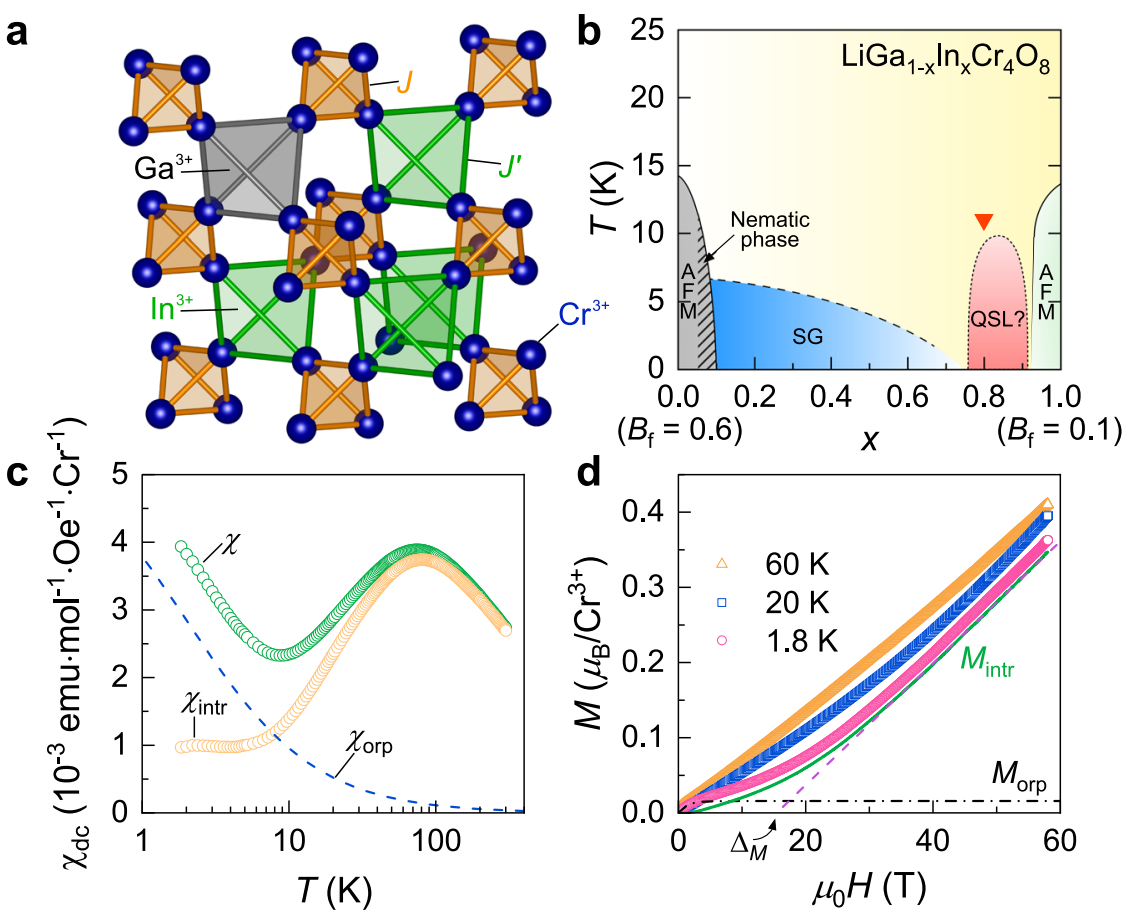

Fig. 1 Crystal structure, phase diagram, and magnetic properties. a Schematic structure of the bond-disordered breathing pyrochlore $\mathrm{LiGa}_{0.2} \mathrm{In}_{0.8} \mathrm{Cr}_{4} \mathrm{O}_{8}$. b $T-x$ phase diagram of $\mathrm{LiGa}_{1-x} \mathrm{In}_{x} \mathrm{Cr}_{4} \mathrm{O}_{8}$. The inverse red triangle marks the investigated $x=0.8$. c Temperature dependence of the static magnetic susceptibility $X_{\mathrm{dc}}(T)$ and its decomposition into the intrinsic $X_{\text {intr }}(T)$ and the orphan spin contribution $X_{\text {orp }}(T)$. $\mathbf{d}$ High-field magnetization at selected temperatures. The black dash-dotted and green dashed lines indicate the orphan spin magnetization $M_{\text {orp }}(T)$ and the bulk magnetization $M_{\text {intr }}(T)$, respectively. A linear extrapolation gives the spin pseudogap of $\Delta_{M} / k_{B} \sim 20 \mathrm{~K}$.

1b. The magnetic ordering vanishes for $x>0.1$ or $x<0.95$. A spinglass phase occupies a wide range of $x=0.1-0.625$. For the Ga-rich compound $\mathrm{LiGa}_{0.95} \mathrm{In}_{0.05} \mathrm{Cr}_{4} \mathrm{O}_{8}$, the ${ }^{7} \mathrm{Li} \mathrm{NMR}$ and neutron experiments revealed the spin nematic transition at $T_{\mathrm{f}}=11 \mathrm{~K}$, driven by the small bond disorder. In the In-rich range of $x=0.75-0.95$, $\mathrm{LiGa}_{1-x} \mathrm{In}_{x} \mathrm{Cr}_{4} \mathrm{O}_{8}$ exhibits a spin-gap-like behavior with the lack of magnetic ordering and spin freezing down to $2 \mathrm{~K}$. However, little is known about an exact ground state and spin dynamics. Here, we have chosen the $x=0.8$ compounds (marked by the inverse triangle in Fig. 1b) to address this issue.

\section{Magnetic susceptibilities and magnetization}

Figure 1c presents the $T$ dependence of dc magnetic susceptibility $X_{\mathrm{dc}}(T)$ of $x=0.8$. On cooling, $X_{\mathrm{dc}}(T)$ shows a broad hump at around $70 \mathrm{~K}$, and a subsequent upturn below $10 \mathrm{~K}$. Above $100 \mathrm{~K}, X_{\mathrm{dc}}(T)$ follows the Curie-Weiss law with the Curie-Weiss temperature $\Theta_{\mathrm{CW}}=-386(1) \mathrm{K}$ and the effective magnetic moment $\mu_{\mathrm{eff}}=3.873$ (4) $\mu_{\mathrm{B}}$ [see Supplementary Note 1] that are consistent with the previously reported values ${ }^{21}$. As the first step to single out the intrinsic $X_{\text {intr }}(T)$, we attempted to fit the $T<5 \mathrm{~K}$ data to $X_{\mathrm{dc}}(T)=$ $X_{\text {intr }}(T)+X_{\text {orp }}(T)$, where $X_{\text {orp }}(T)=C_{\text {orp }} /\left(T-\Theta_{\text {orp }}\right)$. We obtain $\Theta=-2.0$ (2) $\mathrm{K}$, alluding to the contribution of weakly interacting orphan spins. The orphan spin concentration is estimated to be $0.6 \%$ of the $\mathrm{Cr}^{3+}$ spins. Defects and chemical disorders induced by the chemical substitution may be responsible for the appearance of a tiny fraction of the weakly interacting orphan spins. The resulting $X_{\text {intr }}(T<5 \mathrm{~K})$, which is nearly $T$-independent, indicates the presence of abundant low-energy in-gap states. Besides, the ac magnetic susceptibility $X^{\prime}{ }_{\mathrm{ac}}(T)$ excludes the formation of spin freezing or spin glass state [see Supplementary Note 2].

Figure 1d displays the high-field magnetization $M(T, B)$ measured up to $58 \mathrm{~T}$ at selected temperatures. With increasing field, $M(T=1.8 \mathrm{~K}, B)$ shows an upward convex behavior at low fields and then switches to a concave curvature at high fields. The low- $B$ convex $M(B)$ is described by a Brillouin function $B_{J}$, yielding
$1.5 \%$ of the orphan spins. By subtracting $M_{\text {orp }}(B)$ from $M(B)$, we obtain the intrinsic magnetization $M_{\text {intr }}(B)$, as illustrated by the green line in Fig. 1d. The linear extrapolation of the linearly increasing $M_{\text {intr }}(B)$ above 30 T yields the spin pseudogap $\Delta_{M} / k_{B} \sim$ $20 \mathrm{~K}$, comparable to the previous result ${ }^{22}$. The finite slope of $M_{\text {intr }}(B)$ at low fields is associated with the in-gap state, consistent with the $X_{\mathrm{dc}}(T)$ and $X_{\text {ac }}^{\prime}(T)$ [see Supplementary Note 2]. At $T=60 \mathrm{~K}$ $\left(\gg \Delta_{M} / k_{B}\right), M(B)$ shows a quasilinear increase, characteristic of antiferromagnetically coupled spins.

\section{Heat capacity}

In Fig. 2, we plot the heat capacity $C_{\text {tot }}$ of $x=0.8$ as a function of $T$ and $B$. We subtract lattice and nuclear Schottky contributions from $C_{\text {tot }}$ to isolate the magnetic specific heat $C_{\mathrm{m}}$ [see Supplementary Note 3 for details]. As evident from Fig. $2 a, C_{m}$ shows a broad maximum around $70 \mathrm{~K}$, indicative of the onset of short-range ordering, and is largely quenched above $150 \mathrm{~K}$.

From a log-log plot of $C_{m} / T$ vs. $T$ in Fig. 2b, we observe a hump at around $T=0.6 \mathrm{~K}$ in addition to the high- $T$ broad hump. On the application of an external magnetic field, the $T=0.6 \mathrm{~K}$ hump shifts to a higher temperature and is quickly suppressed at $\mu_{0} H>3 \mathrm{~T}$. The $B$-dependence of the low- $T$ hump is attributed to the weakly coupled orphan spins, which occupy the lowest $m_{s}$ level by thermal population. Note that the spin entropy $S_{m}$ has a value of $0.186 \mathrm{~J} \cdot \mathrm{K}^{-1} \cdot \mathrm{mol}^{-1} \cdot \mathrm{Cr}^{-1}$ at $0.6 \mathrm{~K}$, which amounts to roughly $0.2 \%$ of Rln4 [see Supplementary Note 3 for details]. This value is somewhat smaller than the estimated value from $X_{\mathrm{dc}}(T)$ and $M(T=1.8 \mathrm{~K}, B)$.

In addition, $C_{m}(T) / T$ displays a moderate $B$ dependence in the $T$ range of $T=0.1-10 \mathrm{~K}$. Overall, the $C_{\mathrm{m}}(T) / T$ data are described by the power-law dependence $C_{\mathrm{m}} / T \sim T^{n}$ with $n=0.68-0.82$ at $T=$ $2-10 \mathrm{~K}$ and $n=0.67-0.86$ below $1 \mathrm{~K}$. The observed $C_{\mathrm{m}} \sim T^{1.68-1.82}$ is close to a quadratic dependence. We recall that the $T^{2}$ dependence of $C_{m}$ has been reported in a range of 3D frustrated magnets, which are commonly proximate to a disordered QSL 
a

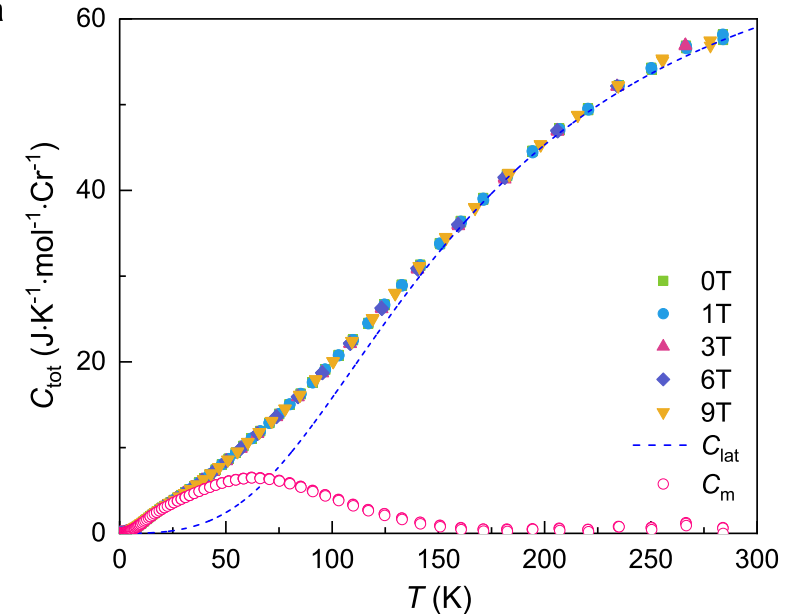

b

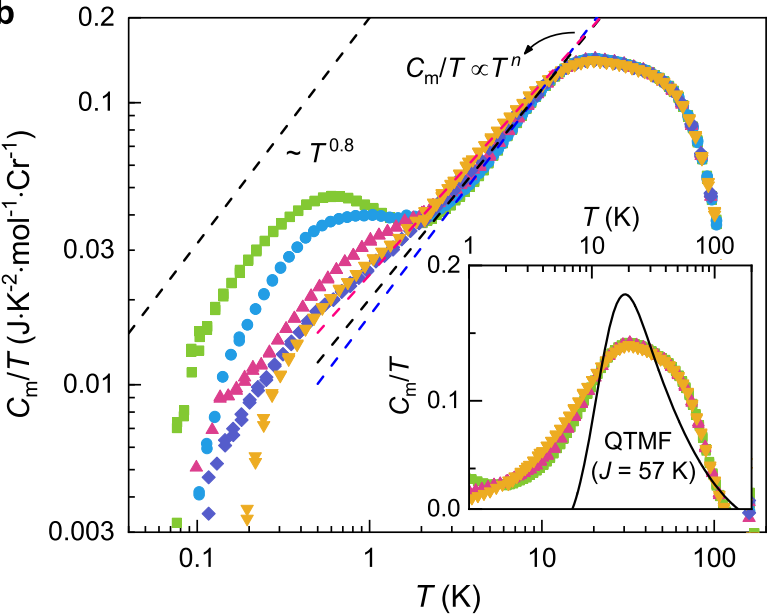

Fig. 2 Heat capacity of the breathing pyrochlore $\mathrm{LiGa}_{0.2}$ $\mathbf{I n}_{\mathbf{0 . 8}} \mathbf{C r}_{\mathbf{4}} \mathbf{O}_{\mathbf{8}}$. a Temperature dependence of the total heat capacity $C_{\text {tot }}$ in various magnetic fields $B=0,1,3,6$, and $9 \mathrm{~T}$. The blue dashed curve denotes the evaluated lattice contributions using the Debye model. The open pink circles represent the magnetic heat capacity $C_{\mathrm{m}} . \mathbf{b} T$ and $B$ dependence of the magnetic heat capacity divided by temperature $C_{\mathrm{m}} / T$ on a double log scale. The dashed lines are fit to the power-law behavior $C_{\mathrm{m}} / T \sim T^{n}$ with $n=0.68-0.82$. The inset shows a comparison between the $C_{\mathrm{m}} / T$ data and the theoretical curve for a $s=3 / 2$ tetrahedron model.

with unconventional low-energy excitations ${ }^{23-25}$. A comparison between the $C_{m} / T$ data and the calculated curve of a $s=3 / 2$ isolated tetrahedron unveils a large discrepancy for temperatures below $10 \mathrm{~K}$ [see the inset of Fig. 2b]. Furthermore, the spin gap is evaluated to be $\Delta / k_{\mathrm{B}}=0.289(5) \mathrm{K}$ from the low- $T$ heat capacity data, suggesting that the excitation gap by tetramer singlets is filled by the in-gap magnetic states at low temperatures [see Supplementary Note 3 for details]. Thus, we conclude that the observed quadratic dependence of $C_{m}$ is associated with emergent gapless low-energy excitations of the bond-disordered breathing pyrochlore and that the orphan spin contribution is minor in forming a spin-liquid-like state, judging from the fact that the gapless excitations develop at temperature as high as $10 \mathrm{~K}$, well above $T=0.6 \mathrm{~K}$.

\section{Nuclear magnetic resonance}

We now examine temporal and thermal spin fluctuations by combining multiple magnetic resonance techniques.

The ${ }^{7} \mathrm{Li}$ NMR spectra and the spin-lattice (spin-spin) relaxation rate $1 / T_{1}\left(1 / T_{2}\right)$ measured at $\mu_{0} H=11.99 \mathrm{~T}$ are plotted as a function of temperature in Fig. 3a, e. At high temperatures, the NMR spectrum exhibits a single sharp line with no quadrupolar splitting, similarly observed in $\mathrm{LilnCr}_{4} \mathrm{O}_{8}{ }^{20,26}$. On cooling down to $T=2.7 \mathrm{~K}$, the NMR spectra gradually broaden and shift to higher magnetic fields without developing additional structured peaks, further confirming the absence of long-range magnetic ordering and structural phase transition.

With decreasing temperature, $1 / T_{1}$ displays an exponential-like decrease down to $10 \mathrm{~K}$, and then a subsequent upturn to a powerlaw growth. The activation behavior of $1 / T_{1}$ in the $T$ range of $25-100 \mathrm{~K}$ is well fitted with the Arrhenius equation $1 / T_{1} \sim \exp (-\Delta /$ $k_{B} T$ ) [see the semi-log plot of $1 / T_{1}$ vs. $1 / T$ in the inset of Fig. 3e]. We obtain the spin pseudogap $\Delta / k_{\mathrm{B}}=35(1) \mathrm{K}$, somewhat larger than $\Delta_{\mathrm{M}} / k_{\mathrm{B}} \sim 20 \mathrm{~K}$ extracted from $M_{\text {intr }}(B)$. Generally, the spin gap $\Delta / k_{\mathrm{B}}$ obtained from $1 / T_{1}$ is larger than the true spin gap $\Delta_{\min }(q)$ occurring at a specific point $q^{27-29}$. This is because $1 / T_{1}$ maps out the $q$-average of the dynamical spin susceptibility, $1 / T_{1} \sim T \Sigma_{q} A^{2}(q)$ $\chi^{\prime \prime}\left(q, \omega_{0}\right)$. Here, $A(q)$ is the form factor of hyperfine interactions, and $\omega_{0}$ is the nuclear Larmor frequency.

The salient feature is that $1 / T_{1}$ follows a power law $1 / T_{1} \sim T^{-n}$ with the exponent of $n=0.46(3)$ below $T^{*}=10 \mathrm{~K}$. The drastically distinct behavior of $1 / T_{1}$ through $T^{*}=10 \mathrm{~K}$ suggests the switching of a dominant relaxation mechanism: a high- $T$ activated vs. a low- $T$ fast fluctuating. Here the small exponent $n$ is indicative of dynamically fluctuating spin excitations, defying the development of a $\lambda$-like critical slowing down. The spin-spin relaxation rate $1 / T_{2}$ shows a weak power-law decrease $1 / T_{2} \sim T^{0.071(4)}$ as $T \rightarrow 0$. A nearly $T$-independent $1 / T_{2}$ means that spin dynamics is in the fastfluctuating limit without critical slowing down, as observed in the QSL states of the triangular material $1 \mathrm{~T}-\mathrm{TaS}_{2}{ }^{30}$.

\section{Muon spin relaxation $(\mu \mathrm{SR})$}

In Fig. 3b, we present ZF- $\mu$ SR spectra at selected temperatures. At high temperatures, the ZF- $\mu$ SR spectra show a Gaussian-like shape and gradually change to an exponential-like form. With decreasing temperature, the muon spin polarization rapidly relaxes in the initial time interval $(t=0-2 \mu \mathrm{s})$. We observe neither an oscillating muon signal down to $T=25 \mathrm{mK}$ nor a recovery to $1 / 3$ of the initial polarization, evidencing the formation of a dynamically fluctuating state $^{31}$. No loss of the initial polarization is not compatible with inhomogeneous magnetism.

For quantitative analysis, we fitted the ZF- $\mu$ SR spectra to the sum of a stretched exponential function and an exponential function, $P_{z}(t)=a_{\text {fast }} \exp \left[-\left(\lambda_{\mathrm{f}} t\right)^{\beta}\right]+a_{\text {slow }} \exp \left[-\lambda_{\mathrm{s}} t\right]$. Here, $a_{\text {fast }}\left(a_{\text {slow }}\right)$ is the fraction of the fast (slow) relaxation component, and $\lambda_{f}\left(\lambda_{s}\right)$ is the muon spin relaxation rate for the fast (slow) relaxing component. We summarize the obtained parameters in Fig. 3f and Supplementary Note 4. As the temperature decreases, the slow relaxation component $\lambda_{s}(T)$ suddenly appears below $T^{*}=10 \mathrm{~K}$, at which ${ }^{7} \mathrm{Li} 1 / T_{1}$ shows an upturn to the power law. Furthermore, $\lambda_{s}(T)$ shows a $T$-independent behavior $\left(\lambda_{\mathrm{s}} \sim 0.042 \mu \mathrm{s}^{-1}\right)$ below $1 \mathrm{~K}$.

Figure $3 \mathrm{f}$ is a log-log plot of the fast relaxation rate vs. $T$. With lowering the temperature, $\lambda_{f}(T)$ exhibits a weak power-law increase $T^{0.11(2)}$ down to $10 \mathrm{~K}$, and then a steep power-law increase with the exponent of $n=-0.71(6)$. Noteworthy is that $\mu \mathrm{SR}$ probes weakly correlated spins in the $T>10 \mathrm{~K}$ range where NMR senses gapped spin excitations, implying the variation of spin dynamics with a detecting time scale. We further note that the steep increment of $\lambda_{\mathrm{f}}(T)$ concurs with the leveling-off of $X_{\text {intr }}(T)$ in Fig. $1 \mathrm{C}$ and the upturn of $1 / T_{1}$ in Fig. 3e. Upon further cooling toward $T=25 \mathrm{mK}, \lambda_{\mathrm{f}}(T)$ flattens out below $2 \mathrm{~K}$, entering a persistent-spin-dynamical regime. Such a leveling-off of the muon spin relaxation rate is often observed in an assortment of QSL candidates $^{32-34}$. With decreasing temperature, the stretched exponent $\beta$ gradually decreases from $\beta=2$ (a Gaussian-like decay) to $\beta=1$ (an exponential decay) below $T^{*}=10 \mathrm{~K}$ [see Supplementary Note 4, Fig. S4]. The low- $T$ exponential relaxation suggests the 
a
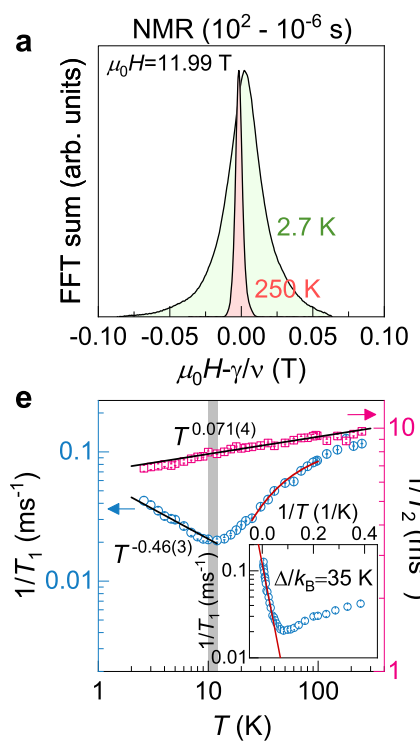
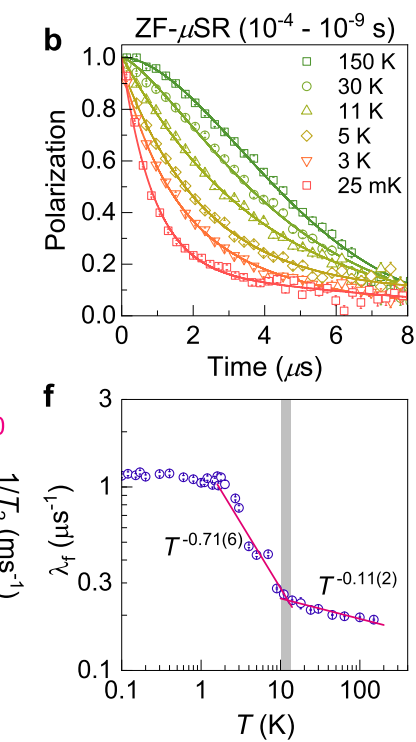
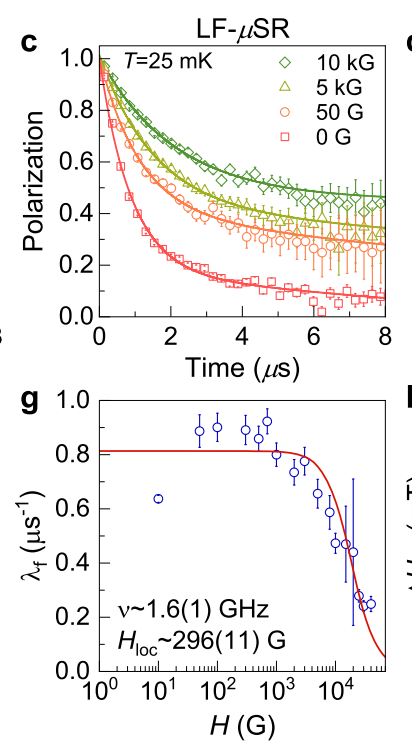
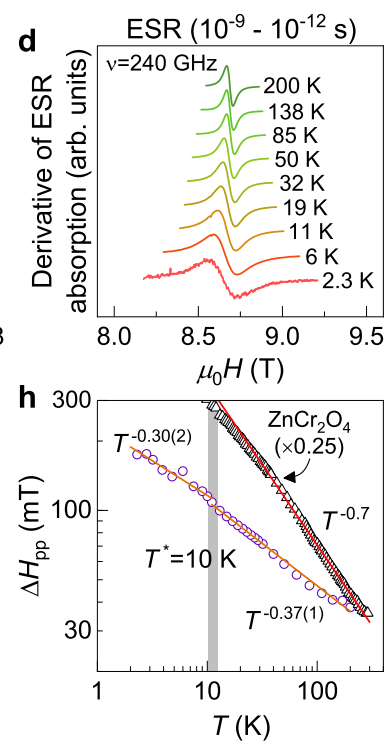

Fig. 3 Thermal and temporal evolution of spin fluctuations of $\mathrm{LiGa}_{0.2} \mathrm{In}_{0.8} \mathrm{Cr}_{4} \mathrm{O}_{8}$ measured by ${ }^{7} \mathrm{Li} \mathrm{NMR}$, ZF- $\mu$ SR, and ESR. a ${ }^{7} \mathrm{Li} \mathrm{NMR} \mathrm{spectra}$ at selected temperatures $T=2.7$ and $250 \mathrm{~K}$. b ZF- $\mu$ SR spectra at selected temperatures. c Longitudinal-field dependence of the $\mu$ SR spectra measured at $T=25 \mathrm{mK}$. The solid lines denote the fit to the data using the sum of two simple exponential functions. $\mathbf{d}$ Derivative of the ESR absorption spectra measured at various temperatures. Spectra are vertically shifted for clarity. e $T$ dependence of ${ }^{7} \mathrm{Li}$ spin-lattice relaxation rate $1 / T_{1}$ and spin-spin relaxation rate $1 / T_{2}$. The red solid line denotes the Arrhenius equation, yielding the spin pseudogap of $\Delta / k_{\mathrm{B}}=35(1) \mathrm{K}$. The solid black line represents a power-law behavior of $1 / T_{1}$ and $1 / T_{2}$. f Muon spin relaxation rate as a function of temperature $\lambda_{f}(T)$. The solid magenta line represents the power-law fit to the data. $\mathbf{g}$ Muon spin relaxation as a function of longitudinal field $\lambda_{\mathrm{LF}}(H)$, along with fitting to the Redfield formula. $\mathbf{h} T$ dependence of the peak-to-peak ESR linewidth $\Delta H_{\mathrm{pp}}(T)$. The solid orange lines indicate a power-law behavior of $\Delta H_{\mathrm{pp}}(T)$ in two different temperature ranges marked by the shaded region at $T=10 \mathrm{~K}$. Error bars on the data points indicate a standard error.

a

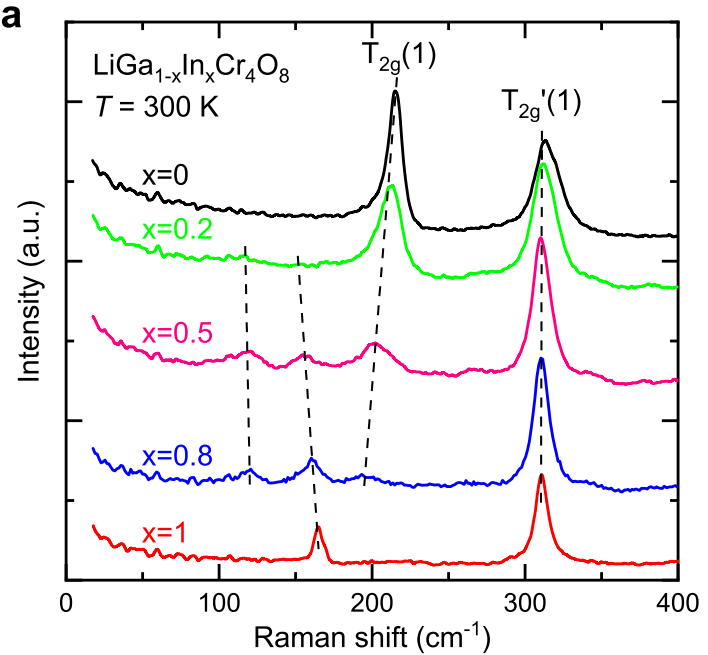

b

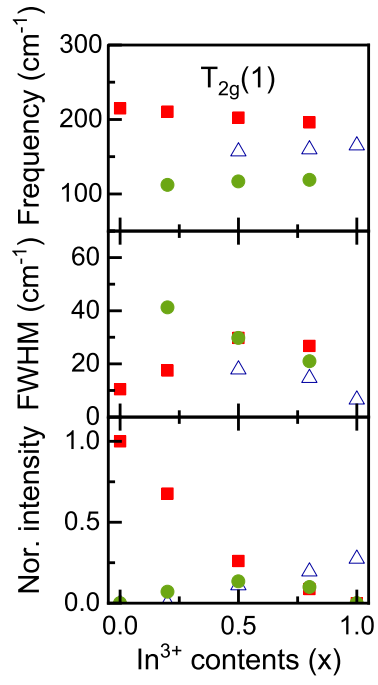

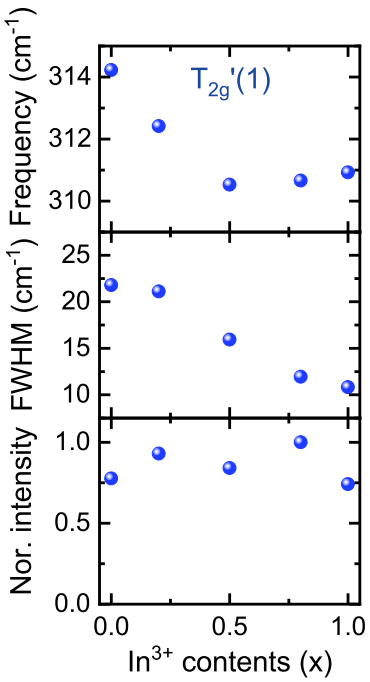

Fig. 4 Comparison of the room-temperature low-energy Raman spectra for $\mathrm{LiGa}_{1-x} \operatorname{In}_{\mathbf{x}} \mathrm{Cr}_{\mathbf{4}} \mathrm{O}_{\mathbf{8}}$. a Composition dependence of the $\mathrm{T}_{2 \mathrm{~g}}(1)$ and $\mathrm{T}_{2 g}^{\prime}(1)$ phonon modes of $\mathrm{LiGa}_{1-x} \mathrm{In}_{x} \mathrm{Cr}_{4} \mathrm{O}_{8}$ measured at room temperature. The dashed lines are guides to the eye. $\mathbf{b}$ Phonon parameters of the $\mathrm{T}_{2 \mathrm{~g}}(1)$ and $\mathrm{T}_{2 \mathrm{~g}}^{\prime}(1)$ modes as a function of $x$.

persisting spin fluctuations to $T=25 \mathrm{mK}$ without forming frozen moments.

Shown in Fig. $3 c$ and $g$ are the LF- $\mu$ SR spectra and the LF dependence of $\lambda_{\mathrm{f}}\left(H_{\mathrm{LF}}\right)$ measured at $T=25 \mathrm{mK}$. Remarkably, the LF$\mu \mathrm{SR}$ spectra exhibit substantial relaxation even at $H_{\mathrm{LF}}=10 \mathrm{kG}$, lending further support to the dynamic ground state. In the Redfield model, $\lambda_{\mathrm{LF}}(H)=2 \gamma_{\mu}^{2}\left\langle H_{\text {loc }}^{2}\right\rangle v /\left(v^{2}+\gamma_{\mu}^{2} H^{2}\right)$, the LF dependence of $\lambda_{\mathrm{f}}\left(H_{\mathrm{LF}}\right)$ is related to the fluctuation frequency $v$, and the fluctuating time-averaged local field $H_{\text {loc }}^{2}{ }^{35}$. Here, $\gamma_{\mu}$ is the muon gyromagnetic ratio. Fitting to the $\lambda_{\mathrm{f}}\left(H_{\mathrm{LF}}\right)$ data yields $v=1.6(1) \mathrm{GHz}$ and $H_{\text {loc }}=296(11) G$ [see Fig. 3g]. We comment that the fluctuation frequency is faster than the value reported thus far for QSL materials while the magnitude of the local field is rather large, possibly due to a large spin number $s=3 / 2^{32,33}$.

\section{Electron spin resonance}

To probe spin fluctuations on the $\mathrm{GHz}$ time scale, we turn to highfrequency ESR. As shown in Fig. 3d, with decreasing temperature, the ESR spectra broaden continuously down to $2.3 \mathrm{~K}$ and shift to a lower field. We observe no impurity signals, excluding the possibility of the $\mathrm{Ga}^{3+} / \mathrm{In}^{3+}$ substitution-induced phase separation. All the ESR spectra are fitted with a single Lorentzian profile, 

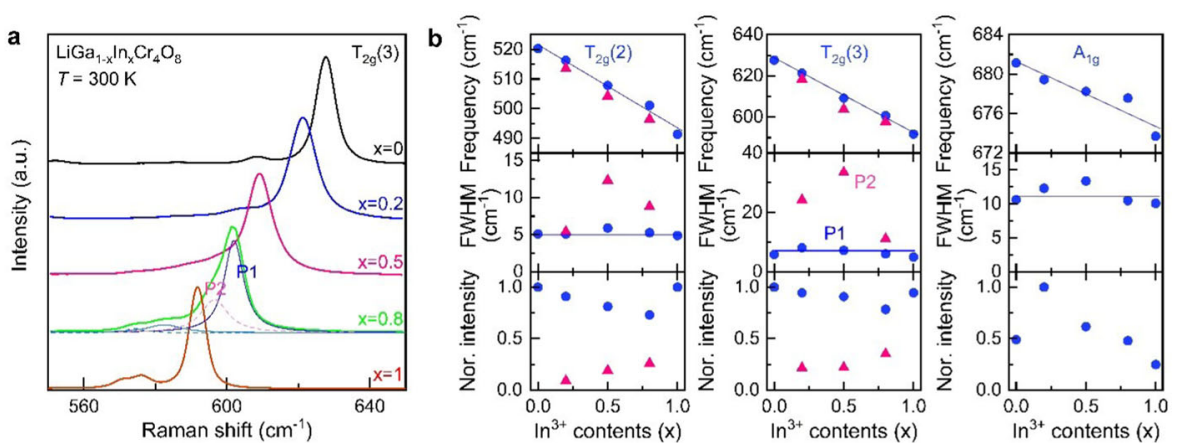

Fig. 5 Composition evolution of high-energy phonons for $\mathrm{LiGa}_{1-x} \mathbf{I n}_{\mathbf{x}} \mathrm{Cr}_{4} \mathbf{O}_{\mathbf{8}}$. a Magnified view of the $\mathrm{T}_{2 g}$ (3) phonon mode as a function of $x$. b Composition dependence of the phonon parameters for the $T_{2 g}(2), T_{2 g}(3)$, and $A_{1 g}$ modes.

suggesting that they originate from one type of the correlated Cr spins. The extracted parameters vs. temperature are plotted in Fig. $3 \mathrm{~h}$ and Supplementary Note 5, Fig. S5. The peak-to-peak linewidth $\Delta H_{\mathrm{pp}}$ is associated with the development of spin-spin correlations, while the resonance field $H_{\text {res }}$ emulates the build-up of internal magnetic fields.

$\Delta H_{\mathrm{pp}}(T)$ exhibits a power-law increase $\Delta H_{\mathrm{pp}}(T) \sim T^{n}$. We can identify a small change of the exponent from $n=0.37(1)$ to $0.30(2)$ through $T^{*}=10 \mathrm{~K}$. This weak anomaly in the exponent is in sharp contrast to the drastically changing character of the magnetic correlations on the $\mathrm{MHz}$ time scale. A critical-like line broadening in the paramagnetic state is due to the development of local spin correlations below $\Theta_{\mathrm{CW}}=-386(1) \mathrm{K}$ and is generic to frustrated magnets subject to critical spin fluctuations. We note that the observed critical exponent of $n=0.30-0.37$ is much smaller than $n=0.6-0.7$ for the uniform pyrochlore $\mathrm{MCr}_{2} \mathrm{O}_{4}$ (for $\mathrm{M}=\mathrm{Zn}, \mathrm{Mg}, \mathrm{Cd})^{36}$, as shown in Fig. 3h. Compared to the uniform pyrochlore, the reduced exponent in $x=0.8$ means that the random bond alternation weakens critical spin correlations. As such, the emergent low- $T$ state in the $\mathrm{GHz}$ time scale bears a resemblance to a diluted pyrochlore lattice.

\section{Raman spectroscopy}

Next, we discuss the structural evolution of $\mathrm{LiGa}_{1-x} \mathrm{In}_{x} \mathrm{Cr}_{4} \mathrm{O}_{8}$ with Raman spectroscopy. We present the composition dependence of the Raman spectra and phonon parameters in Fig. 4. Herein, we focus the $T_{2 g}(1)$ and $T_{2 g}^{\prime}(1)$ phonon modes, which are sensitive to the structural environments of the A and B tetrahedra, respectively [see Supplementary Note 7 and Fig. S7 for the phonon assignments].

As shown in Fig. $4 a, b$, the $314 \mathrm{~cm}^{-1} \mathrm{~T}_{2 g}^{\prime}(1)$ mode slightly softens with increasing $x$ to 0.5 and then little changes with further $\ln ^{3+}$ substitution. Its linewidth decreases linearly with $x$ despite the introduction of $\mathrm{Ga}^{3+} / \mathrm{In}^{3+}$ chemical disorders. This suggests that a single type of $A$ tetrahedra (surrounded by four $\mathrm{Li}^{+}$ ions) is present for all $x$. In sharp contrast, the $T_{2 g}(1)$ mode at $216 \mathrm{~cm}^{-1}$ is split into three peaks for $x=0.5$ and 0.8 . For a random occupation of the $\mathrm{In}^{3+}$ and $\mathrm{Ga}^{3+}$ ions, five types of the $\mathrm{B}$ tetrahedra surrounded by a combination of the $\mathrm{In}^{3+}$ and $\mathrm{Ga}^{3+}$ ions are possible in $\mathrm{LiGa}_{0.2} \mathrm{In}_{0.8} \mathrm{Cr}_{4} \mathrm{O}_{8}$ : four $\mathrm{In}^{3+}(4 \mathrm{l})$, four $\mathrm{Ga}^{3+}(4 \mathrm{G})$, three $\mathrm{In}^{3+}$ /one $\mathrm{Ga}^{3+}(3 \mathrm{IIG}), 2 \mathrm{G} 2 \mathrm{I}$, or $1 \mathrm{G} 3 \mathrm{I}$. For $x=0.8$, the three 118,160 , and $195 \mathrm{~cm}^{-1}$ modes have their scattering intensity in a relative ratio of $1: 1 \cdot 3: 0.7$. Given a low probability of occupying the $3 \mathrm{G} 1 \mathrm{l}$ and the $4 \mathrm{G}$ configurations, the three peaks are assigned to $4 \mathrm{I}, 3 \mathrm{I} 1 \mathrm{G}$, and $2 \mathrm{I} 2 \mathrm{G}$, respectively. From the composition-dependent Raman data, we infer that the $\mathrm{In}^{3+} / \mathrm{Ga}^{3+}$ substitution engenders a single type of the A tetrahedra and three types of the B tetrahedra. However, we admit that the chemical substitution can give rise to diverse effects in addition to the primary chemical pressure, including crystal-electric-field distortion and inhomogeneous charge distribution near the $\mathrm{Cr}^{3+}$ ions. Based solely on the Raman data, it is difficult to rationalize their impact on structure and magnetism. Future high-resolution $\mathrm{x}$-ray studies are required to address this issue.

In Fig. 5a, we zoom the $x$ evolution of the $\mathrm{T}_{2 \mathrm{~g}}(3)$ phonon mode, which involves the asymmetric bending of the $\mathrm{CrO}_{6}$ octahedra. As such, the $\mathrm{T}_{2 \mathrm{~g}}(3)$ mode is sensitive to the substitution-induced alteration of the $\mathrm{Cr}-\mathrm{O}$ bonding geometry, allowing tracing the randomness in exchange interactions. As plotted in Fig. 5b, the phonon frequencies of the $T_{2 g}(2), T_{2 g}(3)$, and $A_{1 g}$ modes show a linear decrease with increasing $x$. A close look at the $T_{2 g}(2)$ and $\mathrm{T}_{2 \mathrm{~g}}(3)$ modes reveals the splitting of the $\mathrm{T}_{2 g}(2)$ and $\mathrm{T}_{2 \mathrm{~g}}(3)$ modes on the $\mathrm{In}^{3+} / \mathrm{Ga}^{3+}$ substitution [see the decomposition of the phonon peaks into four Lorentzian profiles in Fig. 5a and the pink triangles in Fig. 5b]. The linewidths of the main peaks show little variation with $x$. This suggests that $\mathrm{In}^{3+} / \mathrm{Ga}^{3+}$ substitution does not bring about strong disorders such as vacancies, defects, and interstitial cations. Rather, the distinct distortion field induced by the $\mathrm{In}^{3+} / \mathrm{Ga}^{3+}$ substitution leads to the differentiation of $\mathrm{Cr}-\mathrm{O}$ bonding environments. From the observation that most of the high-frequency phonons are split into two peaks, we conclude a differentiation of the intra-tetrahedral $J_{A}$, weak inter-tetrahedral $J_{\mathrm{B} 1}$, and strong inter-tetrahedral exchange interaction $J_{\mathrm{B} 2}$. That is, the bond randomness creates at least exchange interactions $J_{A}, J_{A}^{\prime}$, $J_{B 2}, J_{B 2}^{\prime}, J_{B 1}$, and $J_{B 1}^{\prime}$ with an energy hierarchy $J_{A}, J_{A}^{\prime}>J_{B 2}, J_{B 2}^{\prime}>J_{B 1}$, $J_{B 1}^{\prime}$. Assuming the variations of $\delta J_{A}=J_{A}-J_{A}^{\prime}, \delta J_{B 1}$, and $\delta J_{B 2}$ are not bigger than the $J_{A}$ and $J_{B 2}$, the energy hierarchy is largely preserved.

\section{DISCUSSION}

With the aid of diverse thermodynamic and magnetic resonance techniques $(0 \mathrm{~Hz}-1 \mathrm{THz})$, we are able to disclose the temporal and thermal structures of magnetic correlations.

We identify the characteristic temperature $T^{*}=10 \mathrm{~K}$, below which highly correlated spins develop a quantum paramagnetic state. The emergent low-energy state features nearly $T$-squared $C_{m}$, as well as correlated spin fluctuations over a wide time scale of $10^{-12}-10^{-4} \mathrm{~s}$, as evident from a power-law law $1 / T_{1} \sim T^{-0.46(3)}$, $\lambda_{\mathrm{f}}(T) \sim T^{-0.71(6)}$, and $\Delta H_{\mathrm{pp}}(T) \sim T^{-0.30}$. The exponents extracted from different resonance techniques cannot be related to each other since the breathing pyrochlore model is not analytically solvable. Nonetheless, the common power-law behaviors advocate criticallike spin fluctuations. Here we stress that the energy scale of $T^{*}=$ $10 \mathrm{~K}$ is smaller than the spin pseudogap of $\Delta / k_{\mathrm{B}}=20-35 \mathrm{~K}$, deduced from $M(B)$ and $1 / T_{1}$. Yet, it is bigger than a few kelvins of weakly correlated orphan spins. The latter contribution is quenched by applying the external field of $\mu_{0} H>3 \mathrm{~T}$. As such, the contribution of defect spins to the low- $T$ correlated state is minute.

Next turn to the temporal spin dynamics. The thermodynamic data show the spin-gap behavior with the in-gap state. On the 


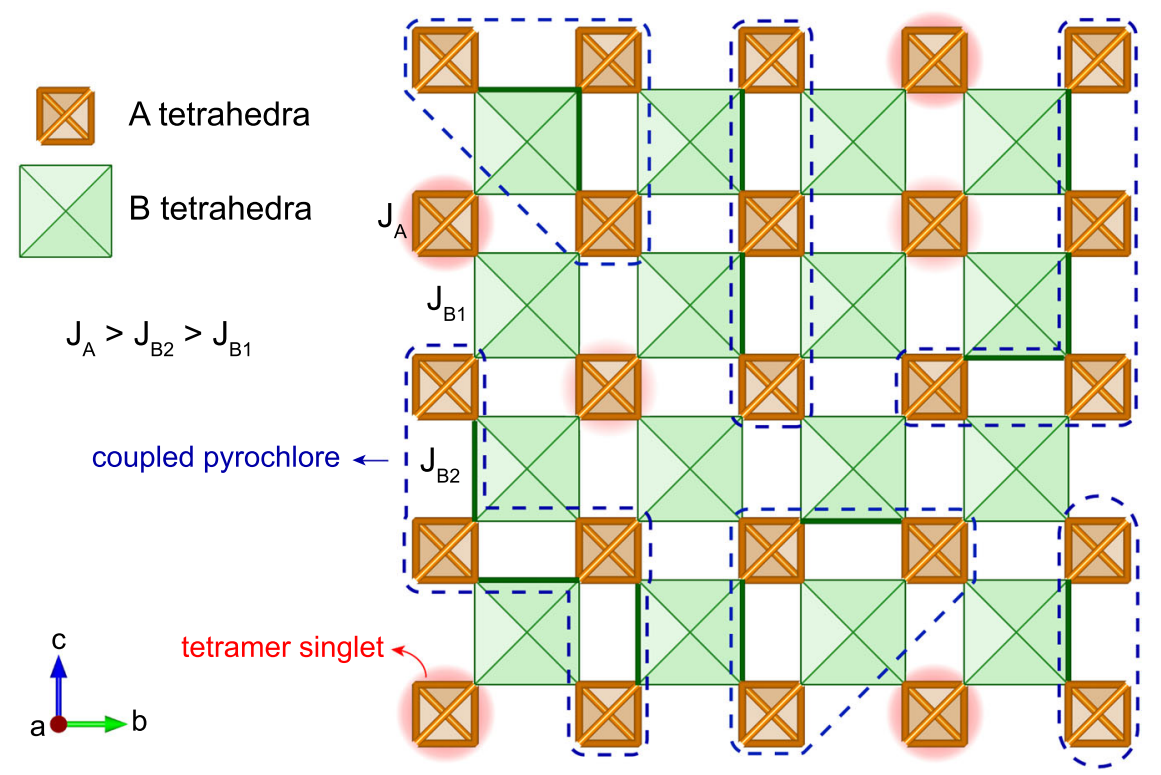

Fig. 6 Illustration of the emergent magnetic lattices in a bond-disordered breathing pyrochlore. Schematics of single-A tetrahedra (orange squares) and three different $B$ tetrahedra (green squares) created by the Ga-for-In substitution. The thick and thin green lines denote $J_{B 2}$ and $J_{B 1}$, respectively. Given the energy hierarchy of magnetic interactions $J_{A}>J_{B 2}>J_{B 1}$, the breathing pyrochlore effectively comprises coupled tetramer singlets (red shaded circle) and diluted pyrochlore lattices (blue dashed region).

slow time scale $\left(1-10^{4} \mathrm{~Hz}\right)$, the NMR data demonstrate dichotomic spin dynamics, namely, high- $T$ gapped spin correlations and low- $T$ fast fluctuations. On the fast time scale $\left(10^{4}-10^{12} \mathrm{~Hz}\right)$, the $\mu \mathrm{SR}$ and ESR data give no signature of the spin-singlet correlations. Instead, highly correlated spin dynamics prevails over the whole measured temperature range. It seems that the singlet fluctuations average out beyond the time scale of the NMR technique at low temperatures.

The dichotomic magnetic correlations are apt to be correlated with structural and magnetic inhomogeneities. First, we mention that the spectral shape and the relaxation rates of the magnetic resonance data rule out the coexistence of magnetically phasesegregated regions. Unlike the end members $(x=0,1), 1 / T_{1}(T)$, $\lambda_{\mathrm{f}}(T)$, and $\Delta H_{\mathrm{pp}}(T)$ of $x=0.8$ show no notable anomalies such as critical divergence, abrupt drop, or kink ${ }^{20,26}$.

Second, the Raman spectra exhibit no phonon anomalies pertaining to magnetostructural transitions [see Supplementary Note 7 for details]. Instead, as sketched in Fig. 6, a statistical distribution of $\mathrm{In}^{3+}$ and $\mathrm{Ga}^{3+}$ generates mainly a single type of $A$ tetrahedra and three types of tetrahedra: four-neighbor $A$ tetrahedra are connected by (i) four weak inter-tetrahedral couplings $J_{B 1} s$, (ii) three weak $J_{B 1} s$ and one strong $J_{B 2}$, and (iii) two weak $J_{B 1} s$ and two strong $J_{B 2} s$. Given the magnetic energy hierarchy $J_{\mathrm{A}}>J_{\mathrm{B} 2}>J_{\mathrm{B} 1}$ with $J_{\mathrm{B} 1} / J_{\mathrm{A}}=0.3$ and $J_{\mathrm{B} 2} / J_{\mathrm{A}}=0.6$, two magnetic subsystems emerge, namely (i) coupled tetramer singlets (red shaded circle) and (ii) diluted pyrochlore lattices (blue dashed region). Here, we note that $J_{\mathrm{B} 1} / J_{\mathrm{A}}$ increases from 0.1 to 0.3 in $\mathrm{LilnCr}_{4} \mathrm{O}_{8}$ through the magnetostructural transition with decreasing temperature ${ }^{37}$. The tetramer singlets forming an entangled state of four spins are coupled through $J_{\mathrm{B} 1}$ and tetrahedral clusters. This bond randomness leads to a broadening and distribution of the discrete energy levels, yet still maintaining a singlet character. Taken together, the coupled tetramer singlets have longer temporal magnetic correlations than the pyrochlorelike subsystem that are governed by two-spin correlations. This raises the possibility that the quantum paramagnetic ground state bears a QSL-like characteristic pertinent in the diluted pyrochlore system. Here, we stress that despite the $\mathrm{In}^{3+} / \mathrm{Ga}^{3+}$ substitutioninduced bond disorders, our thermodynamic and resonance data are not compatible with a spin glass, spin freezing, and random singlets (valence bonds). Of particular, we could find no scalings of thermodynamic quantities expected for random singlets ${ }^{38-40}$. Rather, $\mathrm{LiGa}_{0.2} \mathrm{In}_{0.8} \mathrm{Cr}_{4} \mathrm{O}_{8}$ features a quantum disordered state with gapless low-lying excitations. We, thus, speculate that a degree of randomness is insufficient to engender random singlets or spin glass due to the presence of the tetramer-singlet-like state.

To conclude, we have investigated the temporal and thermal spin dynamics of a bond-disordered breathing pyrochlore compound. We find that a dichotomy of magnetic correlations is linked to two emergent subsystems. $\mathrm{LiGa}_{0.2} \mathrm{In}_{0.8} \mathrm{Cr}_{4} \mathrm{O}_{8}$ occupies a special position in QSL candidate materials because quenched disorders do not lead to a spin glass and random singlet. The observed low-T QSL-like state opens up a venue for discovering an unknown state in a diluted breathing pyrochlore lattice.

\section{METHODS}

\section{Sample synthesis}

Polycrystalline samples of $\mathrm{LiGa}_{0.2} \mathrm{In}_{0.8} \mathrm{Cr}_{4} \mathrm{O}_{8}$ were synthesized by a solidstate reaction method. Stoichiometric amounts of $\mathrm{Li}_{2} \mathrm{CO}_{3}, \mathrm{Cr}_{2} \mathrm{O}_{3}, \mathrm{Ga}_{2} \mathrm{O}_{3}$, and $\ln _{2} \mathrm{O}_{3}$ were mixed in a 1:4:0.2:0.8 molar ratio and thoroughly ground in a mortar. The mixture was pelletized and sintered at $800{ }^{\circ} \mathrm{C}$ in the air for $12 \mathrm{~h}$. The substance was ground, pressured into pellets, and finally sintered at $1000^{\circ} \mathrm{C}$ for $24 \mathrm{~h}$ and $1100^{\circ} \mathrm{C}$ for $72 \mathrm{~h}$. We checked the quality of the samples by X-ray diffraction measurements.

\section{Magnetic properties characterization}

dc and ac magnetic susceptibilities were measured using a superconducting quantum interference device magnetometer and vibrating sample magnetometer (Quantum Design MPMS and VSM). High-field magnetization experiments were performed at the Dresden High Magnetic Field Laboratory using a pulsed-field magnet ( $20 \mathrm{~ms}$ duration). The magnetic moment was detected by a standard inductive method with a pick-up coil system in the field range of $\mu_{0} H=0-60 \mathrm{~T}$.

\section{Heat capacity}

Heat capacity measurements were carried out in the temperature and field range of $T=0.03-300 \mathrm{~K}$ and $B=0-9 \mathrm{~T}$ using a commercial set-up of Quantum Design PPMS with a thermal relaxation method. The raw heat capacity data $C_{\text {tot }} / T$ follow a $T^{-3}$ power-law behavior at extremely low temperatures, corresponding to a nuclear Schottky contribution (see 
Supplementary Note 3). After subtracting the nuclear Schottky contribution $C_{\mathrm{NS}} / T=\mathrm{A} T^{-3}$ from $C_{\mathrm{tot}} / T$, we present the magnetic and lattice contributions to the specific heat in the main text.

\section{Nuclear magnetic resonance}

${ }^{7} \mathrm{Li}\left(I=3 / 2, Y_{\mathrm{N}}=16.5471 \mathrm{MHz} / \mathrm{T}\right) \mathrm{NMR}$ measurements were conducted at National High Magnetic Field Laboratory (Tallahassee, USA) by using a locally developed NMR spectrometer equipped with a high-homogeneity $17 \mathrm{~T}$ sweepable magnet. ${ }^{7} \mathrm{Li}$ NMR spectra were recorded by a fast Fourier transform of spin-echo signals while sweeping the field at a fixed frequency $v=198.317 \mathrm{MHz}$. The nuclear spin-lattice (spin-spin) relaxation time $T_{1}\left(T_{2}\right)$ was measured by a modified inversion recovery (Hahn pulse) method width $\pi / 2=1 \mu \mathrm{s}$ in the temperature range of $T=2.6-250 \mathrm{~K}$.

\section{Muon spin relaxation ( $\mu \mathrm{SR})$}

$\mu \mathrm{SR}$ measurements were carried out on the DR spectrometer $\left({ }^{3} \mathrm{He} /{ }^{4} \mathrm{He}\right.$ dilution refrigerator, $25 \mathrm{mK} \leq T \leq 10 \mathrm{~K}$ ) at $\mathrm{M} 15$ beamline and the LAMPF spectrometer $\left({ }^{4} \mathrm{He}\right.$ cryostat, $\left.2<T<300 \mathrm{~K}\right)$ at M20 beamline in TRIUMF (Vancouver, Canada).

The polycrystalline samples of $\mathrm{LiGa}_{0.2} \mathrm{In}_{0.8} \mathrm{Cr}_{4} \mathrm{O}_{8}$ were wrapped with a silver foil and then attached to the sample holder. After the mounted samples were inserted into the cryostat, $\mu$ SR spectra were measured in zero-field, longitudinal field (parallel to muon spin direction), and transverse field (perpendicular to muon spin direction).

\section{Electron spin resonance (ESR)}

High-frequency ESR experiments were performed at National High Magnetic Field Laboratory (Tallahassee, USA). The ESR spectra were recorded using a quasioptical heterodyne spectrometer in the temperature range of $T=2-290 \mathrm{~K}^{44}$, enabling the detection of a magnetic field derivative of a microwave absorption signal. An external magnetic field was swept with a $12.5 \mathrm{~T}$ sweepable superconducting magnet at the fixed frequency $v=240 \mathrm{GHz}$.

\section{Raman spectroscopy}

The Raman spectra were obtained with a micro-Raman spectrometer (XperRam 200, Nanobase Inc.). Samples were excited in exact backscattering geometry by using a $532 \mathrm{~nm}$ laser.

\section{DATA AVAILABILITY}

The data sets generated and/or analyzed during this study are available from the corresponding author on reasonable request. The $\mu S R$ data can be directly accessed through the TRIUMF Web browser (https://cmms.triumf.ca/mud/).

Received: 18 January 2021; Accepted: 10 April 2021;

Published online: 13 May 2021

\section{REFERENCES}

1. Bramwell, S. T. \& Gingras, M. J. P. Spin ice state in frustrated magnetic pyrochlore materials. Science 294, 1495-1501 (2001).

2. Lee, S.-H. et al. Emergent excitations in a geometrically frustrated magnet. Nature 418, 856-858 (2002).

3. Lee, S.-H. et al. Frustrated magnetism and cooperative phase transitions in spinels. J. Phys. Soc. Jpn. 79, 011004 (2010).

4. Hallas, A. M., Gaudet, J. \& Gaulin, B. D. Experimental insights into ground-state selection of quantum XY pyrochlores. Annu. Rev. Condens. Matter Phys. 9, 105-124 (2018).

5. Rau, J. G. \& Gingras, M. J. P. Frustrated quantum rare-earth pyrochlores. Annu. Rev. Condens. Matter Phys. 10, 357-386 (2019).

6. Bai, X. et al. Magnetic excitations of the classical spin liquid $\mathrm{MgCr}_{2} \mathrm{O}_{4}$. Phys. Rev. Lett. 122, 097201 (2019).

7. Moessner, R. \& Chalker, J. T. Properties of a classical spin liquid: the Heisenberg pyrochlore antiferromagnet. Phys. Rev. Lett. 80, 2929-2932 (1998).

8. Canals, B. \& Lacroix, C. Pyrochlore antiferromagnet: a three-dimensional quantum spin liquid. Phys. Rev. Lett. 80, 2933-2936 (1998).

9. Tsunetsugu, $\mathrm{H}$. Antiferromagnetic quantum spins on the pyrochlore lattice. $J$. Phys. Soc. Jpn. 70, 640-643 (2001).
10. Bramwell, S. T., Gingras, M. J. P. \& Reimers, J. N. Order by disorder in an anisotropic pyrochlore lattice antiferromagnet. J. Appl. Phys. 75, 5523-5525 (1994).

11. Bergman, D. L., Shindou, R., Fiete, G. A. \& Balents, L. Models of degeneracy breaking in pyrochlore antiferromagnets. Phys. Rev. B 74, 134409 (2006).

12. Bellier-Castella, L., Gingras, M. J., Holdsworth, P. C. \& Moessner, R. Frustrated order by disorder: the pyrochlore anti-ferromagnet with bond disorder. Can. J. Phys. 79, 1365-1371 (2001).

13. Tchernyshyov, O., Moessner, R. \& Sondhi, S. L. Order by distortion and string modes in pyrochlore antiferromagnets. Phys. Rev. Lett. 88, 067203 (2002).

14. Kimura, K., Nakatsuji, S. \& Kimura, T. Experimental realization of a quantum breathing pyrochlore antiferromagnet. Phys. Rev. B 90, 060414 (2014).

15. Okamoto, Y., Nilsen, G. J., Attfield, J. P. \& Hiroi, Z. Breathing pyrochlore lattice realized in A-Site ordered spinel oxides $\mathrm{LiGaCr}_{4} \mathrm{O}_{8}$ and $\mathrm{LilnCr}_{4} \mathrm{O}_{8}$. Phys. Rev. Lett. 110, 097203 (2013)

16. Park, S.-Y. et al. Spin-orbit coupled molecular quantum magnetism realized in inorganic solid. Nat. Commun. 7, 12912 (2016).

17. Savary, L. et al. Quantum spin ice on the breathing pyrochlore lattice. Phys. Rev. $B$ 94, 075146 (2016).

18. Li, F.-Y. et al. Weyl magnons in breathing pyrochlore antiferromagnets. Nat. Commun. 7, 12691 (2016).

19. Yan, H., Benton, O., Jaubert, L. D. C. \& Shannon, N. Rank-2 U(1) spin liquid on the breathing pyrochlore lattice. Phys. Rev. Lett. 124, 127203 (2020).

20. Lee, S. et al. Multistage symmetry breaking in the breathing pyrochlore lattice $\mathrm{Li}$ (Ga, In) $\mathrm{Cr}_{4} \mathrm{O}_{8}$. Phys. Rev. B 93, 174402 (2016).

21. Okamoto, Y., Nilsen, G. J., Nakazono, T. \& Hiroi, Z. Magnetic phase diagram of the breathing pyrochlore antiferromagnet $\mathrm{LiGa}_{1-x} \ln \times \mathrm{Cr}_{4} \mathrm{O}_{8}$. J. Phys. Soc. Jpn. 84, 043707 (2015)

22. Okamoto, Y. et al. Magnetic transitions under ultrahigh magnetic fields of up to $130 \mathrm{~T}$ in the breathing pyrochlore antiferromagnet $\mathrm{LilnCr}_{4} \mathrm{O}_{8}$. Phys. Rev. B 95 134438 (2017).

23. Okamoto, Y., Nohara, M., Aruga-Katori, H. \& Takagi, H. Spin-liquid state in the $\mathrm{S}=1 / 2$ hyperkagome antiferromagnet $\mathrm{Na}_{4} \mathrm{Ir}_{3} \mathrm{O}_{8}$. Phys. Rev. Lett. 99, 137207 (2007).

24. Silverstein, $\mathrm{H}$. J. et al. Liquidlike correlations in single-crystalline $\mathrm{Y}_{2} \mathrm{Mo}_{2} \mathrm{O}_{7}$ : an unconventional spin glass. Phys. Rev. B 89, 054433 (2014).

25. Plumb, K. W. et al. Continuum of quantum fluctuations in a three-dimensional $\mathrm{S}=1$ Heisenberg magnet. Nat. Phys. 15, 54-59 (2018).

26. Tanaka, Y., Yoshida, M., Takigawa, M., Okamoto, Y. \& Hiroi, Z. Novel phase transitions in the breathing pyrochlore lattice: ${ }^{7} \mathrm{Li}-\mathrm{NMR}$ on $\mathrm{LilnCr}_{4} \mathrm{O}_{8}$ and $\mathrm{LiGaCr}_{4} \mathrm{O}_{8}$ Phys. Rev. Lett. 113, 227204 (2014).

27. Shimizu, T., MacLaughlin, D. E., Hammel, P. C., Thompson, J. D. \& Cheong, S.-W Spin susceptibility and low-lying excitations in the Haldane-gap compound $\mathrm{Y}_{2} \mathrm{BaNiO}_{5}$. Phys. Rev. B 52, R9835-R9838 (1995).

28. Furukawa, Y., Iwai, A., Kumagai, K. \& Yakubovsky, A. ${ }^{31} \mathrm{P}-\mathrm{NMR}$ study of low-energy spin excitations in spin ladder $(\mathrm{VO})_{2} \mathrm{P}_{2} \mathrm{O}_{7}$ and spin dimer $\mathrm{VO}\left(\mathrm{HPO}_{4}\right)_{0.5} \mathrm{H}_{2} \mathrm{O}$ systems. J. Phys. Soc. Jpn. 65, 2393-2396 (1996).

29. Baek, S.-H., Choi, K.-Y., Berger, H., Büchner, B. \& Grafe, H.-J. Persistence of singlet fluctuations in the coupled spin tetrahedra system $\mathrm{Cu}_{2} \mathrm{Te}_{2} \mathrm{O}_{5} \mathrm{Br}_{2}$ revealed by highfield magnetization, ${ }^{79} \mathrm{Br} \mathrm{NQR}$, and ${ }^{125} \mathrm{Te}$ NMR. Phys. Rev. B 86, 180405 (2012).

30. Klanjšek, M. et al. A high-temperature quantum spin liquid with polaron spins. Nat. Phys. 13, 1130-1134 (2017).

31. Uemura, Y. J., Yamazaki, T., Harshman, D. R., Senba, M. \& Ansaldo, E. J. Muon-spin relaxation in AuFe and CuMn spin glasses. Phys. Rev. B 31, 546-563 (1985).

32. Mendels, $P$. et al. Quantum magnetism in the paratacamite family: towards an ideal Kagomé lattice. Phys. Rev. Lett. 98, 077204 (2007).

33. Li, Y. et al. Muon spin relaxation evidence for the $U(1)$ quantum spin-liquid ground state in the triangular antiferromagnet $\mathrm{YbMgGaO}_{4}$. Phys. Rev. Lett. 117, 097201 (2016).

34. Balz, C. et al. Physical realization of a quantum spin liquid based on a complex frustration mechanism. Nat. Phys. 12, 942-949 (2016).

35. Yaouanc, A. \& Réotier, P. D. de. Muon Spin Rotation, Relaxation, and Resonance: Applications to Condensed Matter. (Oxford University Press, 2011).

36. Hemmida, M., Nidda, H.-A. K., von Tsurkan, V. \& Loidl, A. Berezinskii-KosterlitzThouless type scenario in the molecular spin liquid $\mathrm{ACr}_{2} \mathrm{O}_{4}(\mathrm{~A}=\mathrm{Mg}, \mathrm{Zn}, \mathrm{Cd})$. Phys. Rev. B 95, 224101 (2017).

37. Nilsen Gøran, J. et al. Complex magnetostructural order in the frustrated spinel LilnCr ${ }_{4} \mathrm{O}_{8}$. Phys. Rev. B 91, 174435 (2015).

38. Kimchi, I., Nahum, A. \& Senthil, T. Valence bonds in random quantum magnets: theory and application to $\mathrm{YbMgGaO}_{4}$. Phys. Rev. X 8, 031028 (2018).

39. Uematsu, K. \& Kawamura, H. Randomness-induced quantum spin liquid behavio in the $s=1 / 2$ random-bond Heisenberg antiferromagnet on the pyrochlore lattice. Phys. Rev. Lett. 123, 087201 (2019).

40. Kawamura, H. \& Uematsu, K. Nature of the randomness-induced quantum spin liquids in two dimensions. J. Phys. Condens. Matter 31, 504003 (2019).

41. Tol, J., van Brunel, L. C. \& Wylde, R. J. A quasioptical transient electron spin resonance spectrometer operating at 120 and $240 \mathrm{GHz}$. Rev. Sci. Instrum. 76 , 074101 (2005). 


\section{ACKNOWLEDGEMENTS}

We would like to thank B. Hitti for the technical support with $\mu$ SR experiments at TRIUMF and G. Stenning for assistance on the heat capacity measurements in the Materials Characterization Laboratory at the ISIS Neutron and Muon Source. We thank F. C. Chou for the fruitful discussion. This work was supported by the National Research Foundation (NRF) of Korea (Grants No. 2020R1A2C3012367, No. 2020R1A5A1016518, and No. 2013M7A1A1075764). A portion of this work was performed at the National High Magnetic Field Laboratory, which is supported by the National Science Foundation Cooperative Agreement No. DMR-1157490 and No. DMR-1644779 and the State of Florida. We acknowledge the support of the HLD at HZDR, a member of the European Magnetic Field Laboratory (EMFL).

\section{AUTHOR CONTRIBUTIONS}

K.-Y.C. designed and conceived the study. S.L., S.-H.D, and K.-Y.C. planned the experiments. S.-H.D. and Y.S.C. synthesized the sample and characterized structural and magnetic properties. W.L. and A.P.R. conducted NMR experiments. S.L. and W.L. performed $\mu$ SR experiments, and S.L. analyzed the data. S.L. and J.V.T. carried out the high-field ESR measurements. S.L. carried out the heat capacity measurements. D.G. conducted high-field magnetization experiments. Y.S.C. performed the Raman experiments and analyzed the data. S.L. and K.-Y.C. wrote the manuscript with contributions from all authors.

\section{COMPETING INTERESTS}

The authors declare no competing interests.

\section{ADDITIONAL INFORMATION}

Supplementary information The online version contains supplementary material available at https://doi.org/10.1038/s41535-021-00347-0.

Correspondence and requests for materials should be addressed to K.-Y.C.

Reprints and permission information is available at http://www.nature.com/ reprints

Publisher's note Springer Nature remains neutral with regard to jurisdictional claims in published maps and institutional affiliations.

Open Access This article is licensed under a Creative Commons Attribution 4.0 International License, which permits use, sharing, adaptation, distribution and reproduction in any medium or format, as long as you give appropriate credit to the original author(s) and the source, provide a link to the Creative Commons license, and indicate if changes were made. The images or other third party material in this article are included in the article's Creative Commons license, unless indicated otherwise in a credit line to the material. If material is not included in the article's Creative Commons license and your intended use is not permitted by statutory regulation or exceeds the permitted use, you will need to obtain permission directly from the copyright holder. To view a copy of this license, visit http://creativecommons. org/licenses/by/4.0/.

(c) The Author(s) 2021 